\title{
Exogenous gibberellin altered morphology, anatomic and transcriptional regulatory networks of hormones in carrot root and shoot
}

\author{
Guang-Long Wang, Feng Que, Zhi-Sheng Xu, Feng Wang and Ai-Sheng Xiong*
}

\begin{abstract}
Background: Gibberellins stimulate cell elongation and expansion during plant growth and development. Carrot is a root plant with great value and undergoes obvious alteration in organ size over the period of plant growth. However, the roles of gibberellins in carrot remain unclear.

Results: To investigate the effects of gibberelliins on the growth of carrot, we treated carrot plants with gibberellic acid $3\left(\mathrm{GA}_{3}\right)$ or paclobutrazol (a gibberellin inhibitor). The results found that $\mathrm{GA}_{3}$ dramatically reduced the root growth but stimulated the shoot growth of carrot. It also significantly promoted xylem development in the tuberous root of carrot. In addition, transcript levels of genes related to gibberellins, auxin, cytokinins, abscisic acid and brassinolides were altered in response to increased or reduced gibberellins.

Conclusions: The inhibited tuberous root growth but enhanced shoot growth in plants treated with $\mathrm{GA}_{3}$ can be principally attributed to the changes in the xylem development of carrot roots. Negative feedback regulation mechanism of gibberellin biosynthesis also occurred in response to altered gibberellin accumulation. Gibberellins may interact with other hormones to regulate carrot plant growth through crosstalk mechanisms. This study provided novel insights into the functions of gibberellins in the growth and development of carrot.
\end{abstract}

Keywords: Gibberellins, Morphology, Anatomic, Transcript profiles, Hormonal crosstalk, Daucus carota L

\section{Background}

Plant growth and development are stimulated by environmental or intrinsic cues, such as hormones [1, 2]. Gibberellins (GAs) are indispensable stimulators of plant growth [3]. These hormones are initiated from the diterpenoid pathway. To date, over 100 GAs have been identified, but only a few of them are bioactive [4]. Nowadays, the application of exogenous GAs is commonly used to regulate plant growth and development [5].

GA metabolism and perception have been extensively documented [6]. In vascular plants, geranyl geranyl diphosphate (GGDP) is first converted to ent-kaurene by ent-copalyl diphosphate synthase (CPS) and ent-kaurene

\footnotetext{
* Correspondence: xiongaisheng@njau.edu.cn

State Key Laboratory of Crop Genetics and Germplasm Enhancement, College of Horticulture, Nanjing Agricultural University, Nanjing 210095, China
} ent-kaurene oxidase $(\mathrm{KO})$ and ent-kaurenoic acid oxidase (KAO). Stepwise oxidation is catalyzed by GA20oxidase (GA20ox) and GA3-oxidase (GA3ox) to produce various GA intermediates and bioactive GAs, whereas GA2-oxidase (GA2ox) is involved in inactivation of bioactive GAs. GA-mediated growth is attributed to the egradation of DELLA proteins, which are the main restraints for plant growth [7]. Binding of GA to the reptor, GIBBERELLIN INSENSITIVE DWARF1 (GID1) ggers a close link between GID1 and DELLA; subsetly, DELLAs are degraded via the $26 \mathrm{~S}$ proteasome 8]. Other components, such as specific ubiquitin E3

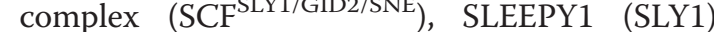
SHORT INTERNODE (SHI) and SPINDLY (SPY), are essentially required for GA signal transduction (Fig. 1) [9]. Biochemical, molecular and genetic 


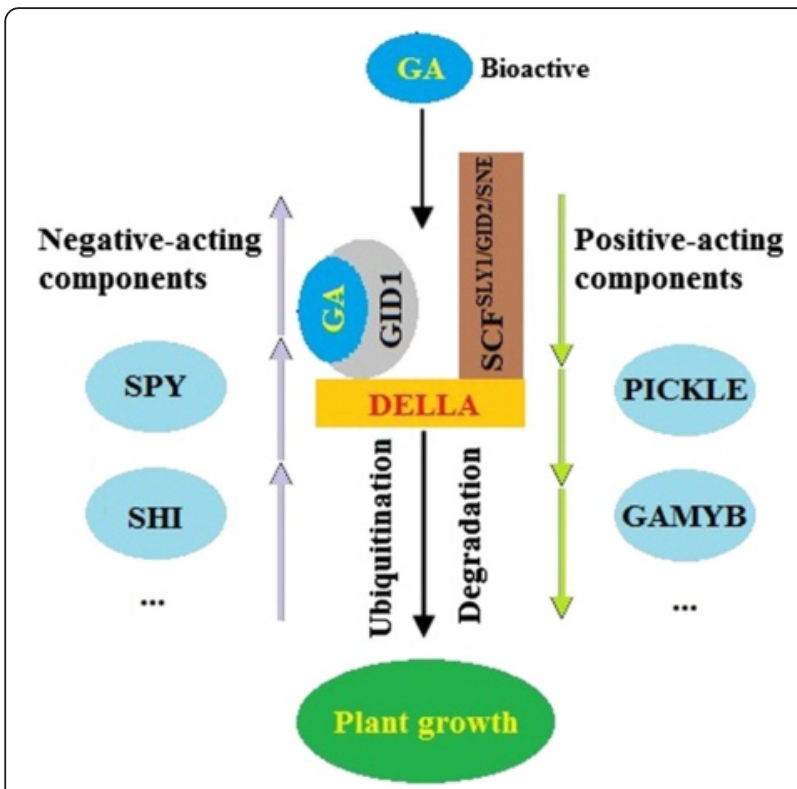

Fig. 1 Receptors and acting components in GA response in higher plants

studies have suggested that genes involved in GA metabolic and signaling pathways are essential for GA accumulation and subsequent functions [10-12].

GAs are involved in various processes in plants. GAdeficient mutants of Arabidopsis and tomato cannot germinate without exogenous GAs [13]. By contrast, GA application can make environmental stimuli unnecessary and promote germination, indicating that GAs play essential roles in germination [14]. GAs can promote cell elongation, and $\mathrm{GA}_{4}$ is the active form that regulates shoot elongation in Arabidopsis [15]. GAs also participate in leaf expansion and fruit set and growth [16, 17]. Furthermore, GAs control root elongation and cell proliferation $[18,19]$.

Carrot (Daucus carota L.), a biennial plant from the Apiaceae family, is a root vegetable with enriched healthy composition [20-22]. Our previous work has focused on conducting carrot breeding and establishing genome and transcriptome database for carrot (http://apiaceae.njau.edu. $\mathrm{cn} /$ carrotdb/index.php) [23]. However, the role of GAs in the regulation of root plants is poorly understood because of technical reasons [24]. Hidden underground, root systems are often difficult to observe and quantify without any harm. A previous research indicated that GA is essentially required for carrot somatic embryogenesis [25]. However, another study suggested that applied GA inhibits the growth of carrot roots [26]. Thus, the functions of GAs in carrot remain unclear.

The present study aimed to investigate the effects of GA treatment on the growth and development of carrot. We attempted to gain novel insights into GA functions in carrot growth based on transcript profiles of genes involved in hormone metabolic and signaling pathways. Morphological and anatomical characteristics, along with hormone crosstalk, were also discussed to completely elucidate the roles of applied $\mathrm{GA}_{3}$. This study provided novel insights into GA-mediated plant growth and development in vascular plants.

\section{Results}

Plant growth analysis

To determine whether GA is involved in carrot plant growth, 5-week-old carrots were treated with GA or its inhibitor paclobutrazol (PBZ) weekly for five times. The effects of the applied GA and PBZ on carrot plant growth were observed after 5 weeks (Fig. 2). Exogenous $\mathrm{GA}_{3}$ significantly increased the shoot weight but significantly decreased root diameter and the root weight of carrot. By contrast, PBZ increased the root weight and root diameter but decreased the shoot weight of carrot. The exogenous application of $\mathrm{GA}_{3}+\mathrm{PBZ}$ resulted in a phenotype similar to that of the control, which was the intermediate between the $\mathrm{GA}_{3}$ and the $\mathrm{PBZ}$ treated plants (Fig. 3).

\section{Anatomical structure changes in the roots, petioles and leaves \\ In the roots}

Carrot roots without any treatment were approximately $1.2 \mathrm{~cm}$ in diameter (Fig. 4a). $\mathrm{GA}_{3}$ dramatically reduced the root diameter, which was slightly relieved in the presence of PBZ (Figs. 3 and 4). Interestingly, the ratio of xylem area to total root area was significantly higher under $\mathrm{GA}_{3}$ treatment compared with control conditions (Fig. 5). PBZ alone significantly increased the thickness of root diameter, but decreased the ratio of xylem area to total area, which was relieved by application of $\mathrm{GA}_{3}$ (Figs. 3, 4 and 5).

\section{In the petioles}

$\mathrm{GA}_{3}$ increased the number of vascular bundles in the petioles, which may contribute to influxes of nutrients and water towards the leaves (Fig. 6b). This effect was also observed when $\mathrm{GA}_{3}$ was applied together with PBZ. However, PBZ did not evidently change the number of vascular bundles (Figs. 6 and 7).

\section{In the leaves}

Palisade tissue $(\mathrm{Pt})$ and spongy tissue $(\mathrm{St})$ are two main components of carrot leaves. Pt, which contains the largest number of chloroplasts, may be the principal site for photosynthesis in the leaves. St is another leaf tissue that facilitates gas exchange. However, no obvious difference was detected in the anatomical structure of the leaves under different treatments (Fig. 8). 


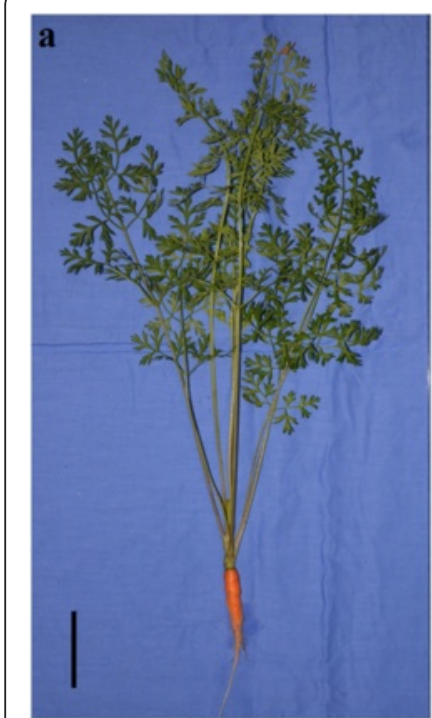

Control

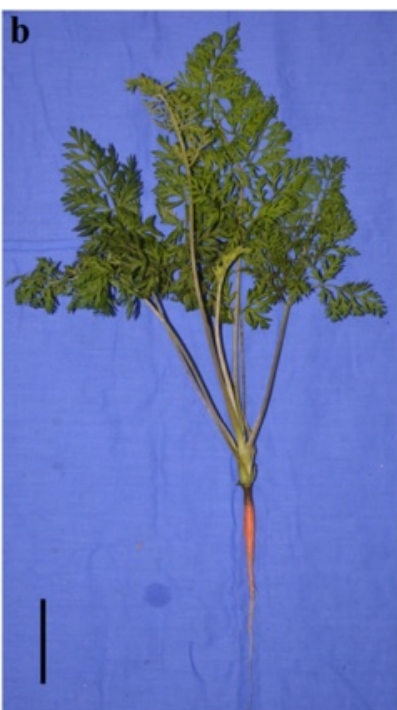

$\mathbf{G A}_{3}$

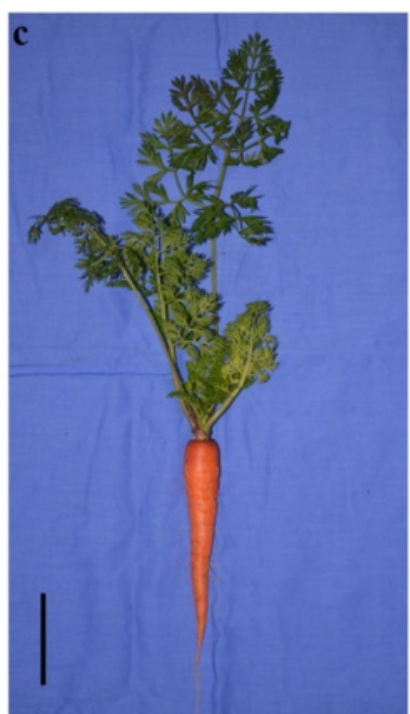

PBZ

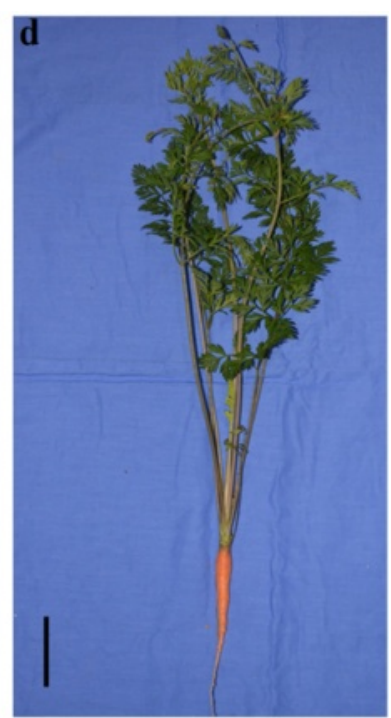

GA ${ }_{3}+\mathbf{P B Z}$

Fig. 2 Effects of $\mathrm{GA}_{3}, \mathrm{PBZ}$ or $\mathrm{GA}_{3}+\mathrm{PBZ}$ on carrot growth. Black lines in the lower left corner of each plant represent $5 \mathrm{~cm}$ in that pixel

Effects of $\mathrm{GA}_{3}$ treatment on the expression levels of $\mathrm{GA}$ biosynthetic pathway genes

To illustrate the effects of gibberellin application on GA biosynthesis, we investigated the changes in the expression levels of GA metabolic genes. DcKS, DcKO, DcKAO1, DcGA20ox1, DcGA20ox2, DcGA3ox1, DcGA2ox1, $D c G A 2 o x 2$ and $D c G A 2 o x 3$ were annotated as GA pathwayrelated genes based on carrotDB, a transcriptomic and genomic database for carrot (Table 1). The expression levels of these selected genes were measured by quantitative real-time PCR (qRT-PCR). The biosynthetic pathway- related genes were strongly regulated by GA or PBZ treatment (Fig. 9).

In the roots, exogenous $\mathrm{GA}_{3}$ application upregulated DcKS, DcGA2ox1, DcGA2ox2 and DcGA2ox3 expression but reduced the mRNA levels of DcKO,DcGA20ox1 and DcGA20ox2. Similarly, $\mathrm{GA}_{3}+$ PBZ treatment markedly decreased the expression levels of DcKO,DcGA20ox1 and $D c G A 20 o x 2$ but obviously increased that of DcGA2ox2. In the petioles, exogenous $\mathrm{GA}_{3}$ application reduced the mRNA levels of DcKS, DcKO, DcKAO1, DcGA2ox1, $D c G A 2 o x 2$ and $D c G A 2 o x 3$ but did not significantly alter

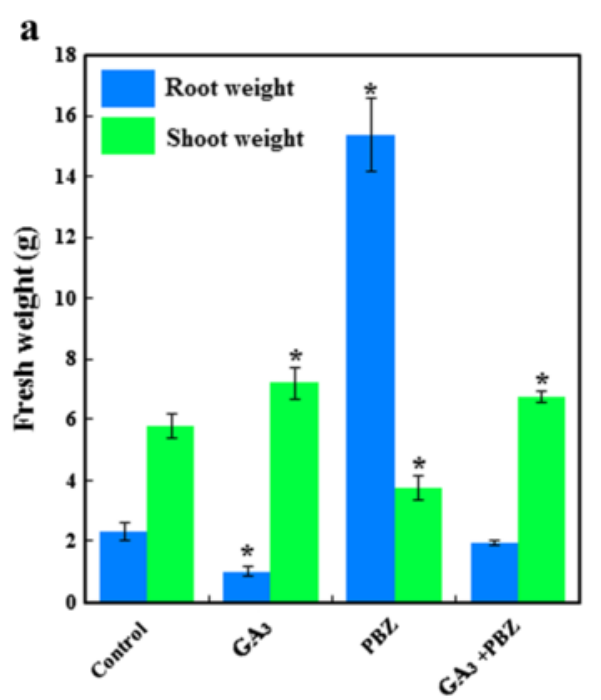

Treatments

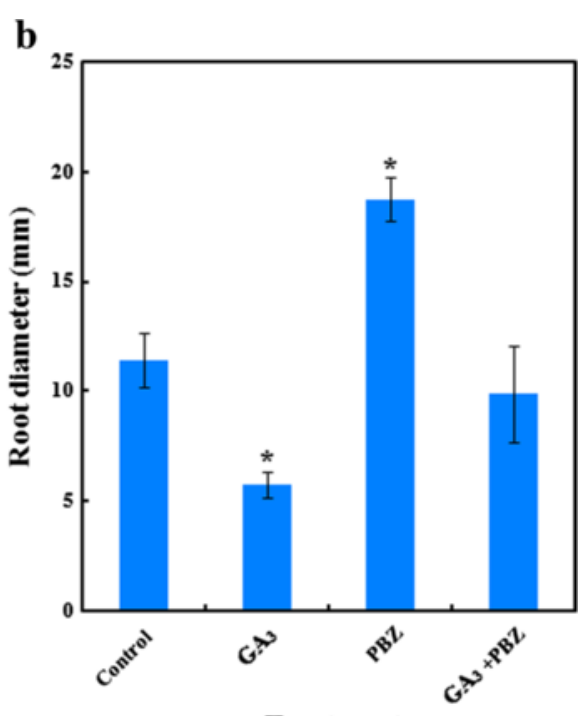

Treatments

Fig. 3 Effects of $\mathrm{GA}_{3}$, PBZ or $\mathrm{GA}_{3}+\mathrm{PBZ}$ on carrot fresh weight (a) and root diameter (b). Values are means \pm SD from three replicates, and the bars represent SD. Statistical differences were evaluated by Student $t$ test at the 0.05 probability level. The asterisk indicates a significant difference compared with control group 


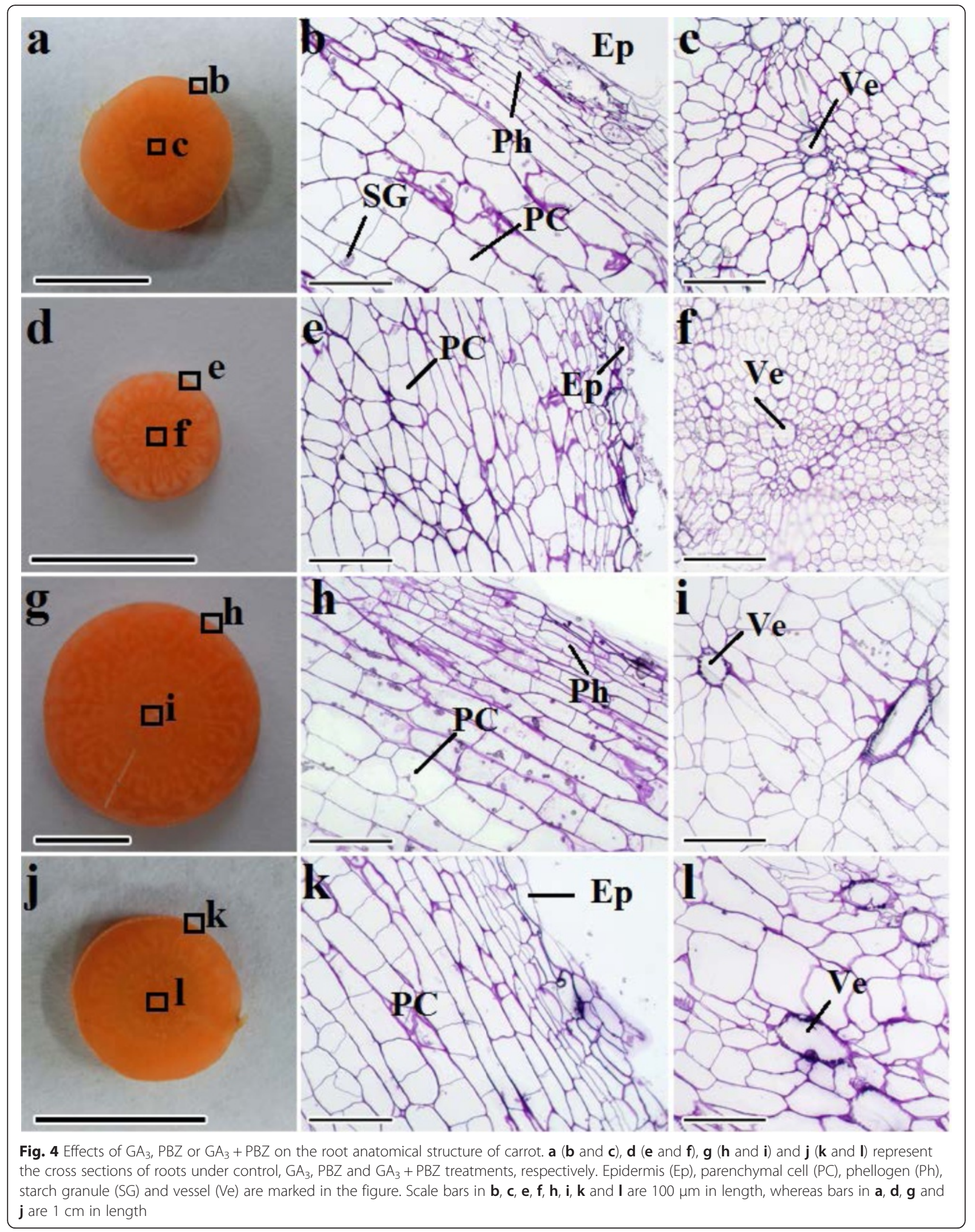




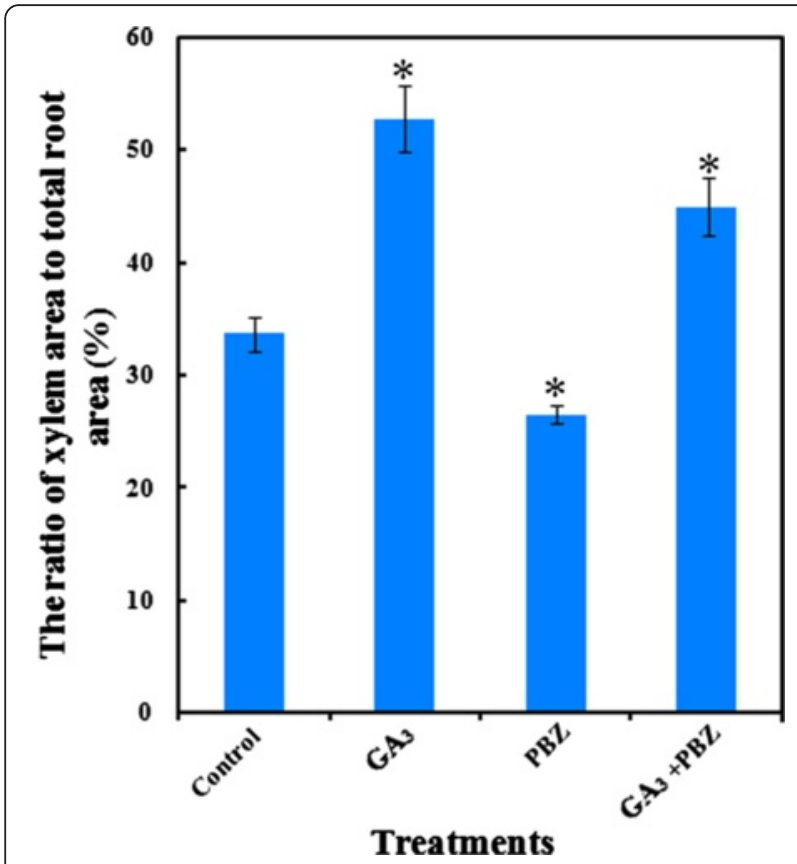

Fig. 5 Effects of $\mathrm{GA}_{3}$ and PBZ treatments on the ratio of xylem area to total root area. Values are means \pm SD from three replicates, and the bars represent SD. Statistical differences were evaluated by Student $t$ test at the 0.05 probability level. The asterisk indicates a significant difference compared with control group the expression levels of DcGA20ox1, DcGA20ox2 and DcGA3ox1. PBZ alone upregulated DcKO, DcKAO1, DcGA20ox1, DcGA20ox2, and DcGA3ox1 but downregulated $D c G A 2 o x 1$ and $D c G A 2 o x 3$. In the leaves, $\mathrm{GA}_{3}$ application upregulated DcGA2ox1 but downregulated DcKS and DcGA20ox2 expression. PBZ alone upregulated DcGA20ox1 but downregulated DcGA2ox2 and DcGA2ox3 (Fig. 9).

Effects of $\mathrm{GA}_{3}$ application on the expression levels of $\mathrm{GA}$ response genes

The proteins encoded by DcGID1b, DcGID1c, DcDELLA, DcSLY1, DcPICKLE1, DcPICKLE2, DcSPY, DcGAMYB and $D c S H I$ were recognized as GA receptors or acting components by carrotDB. Thus, these genes were selected and investigated for qRT-PCR analysis (Fig. 10).

In the roots, $\mathrm{GA}_{3}$ treatment downregulated DcGID1b and DcSLY1 but upregulated DcDELLA, DcSPY and $D c S H I$. In the petioles, $\mathrm{GA}_{3}$ treament alone downregulated DcGID1c and DcPICKLE1. By contrast, DcGID1b, $D c G I D 1 c$ and DcPICKLE1 showed increased expression after PBZ treatment. In the leaves, exogenous $\mathrm{GA}_{3}$ upregulated DELLA and DCSPY. PBZ increased DcGID1c but suppressed DcDELLA (Fig. 10).

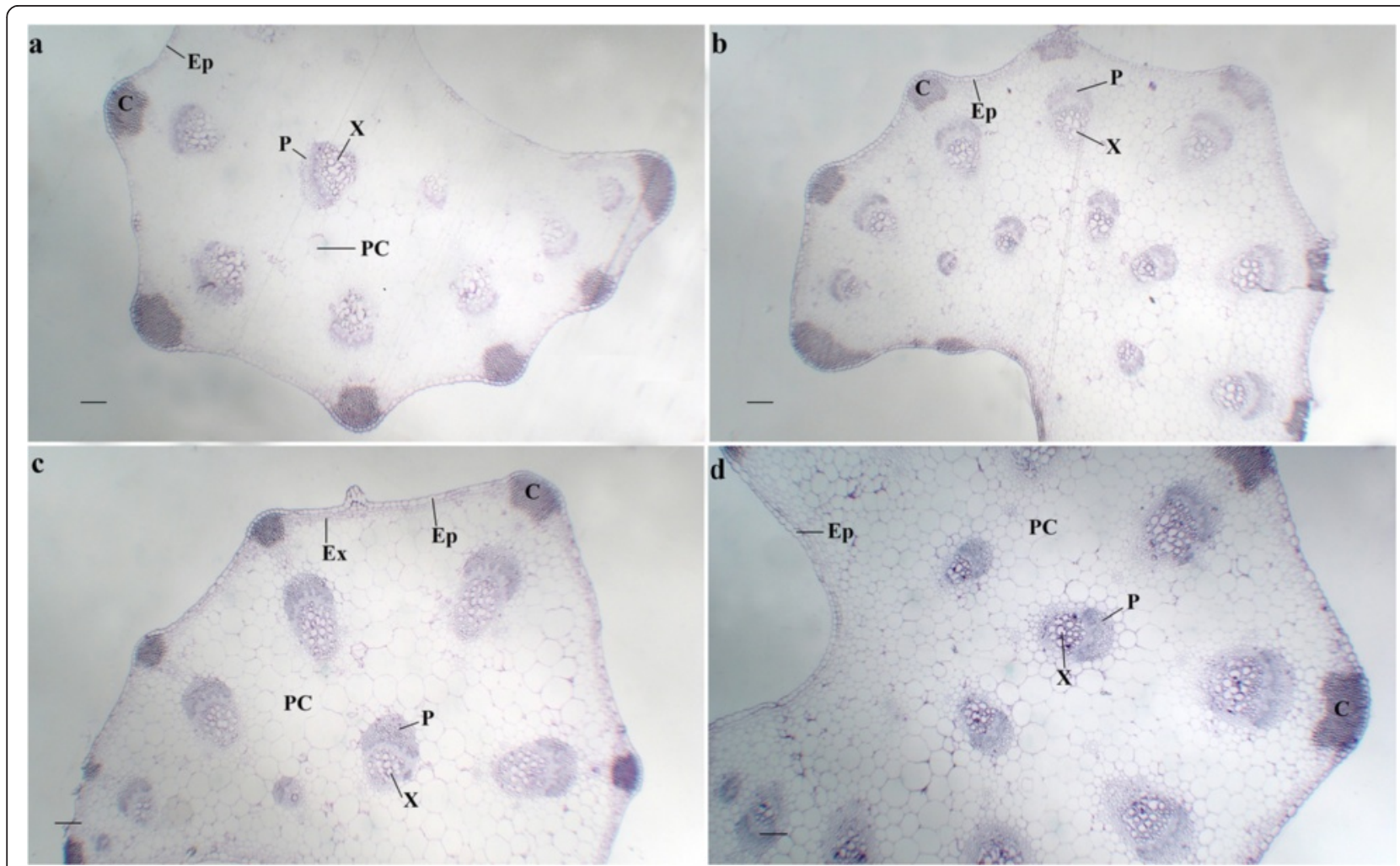

Fig. 6 Effects of $G A_{3}, P B Z$ or $G A_{3}+P B Z$ on the anatomical structure in carrot petioles. $\mathbf{a}, \mathbf{b}, \mathbf{c}$ and $\mathbf{d}$ represent the cross sections of petioles under control, $G A_{3}, P B Z$ and $G A_{3}+P B Z$ treatments, respectively. Collenchyma (C), epidermis (Ep), exodermis (Ex), phloem (P) and xylem (X) are marked in the figure. Scale bars in $\mathbf{a}, \mathbf{b}, \mathbf{c}, \mathbf{d}$ and e are $40 \mu \mathrm{m}$ in length 


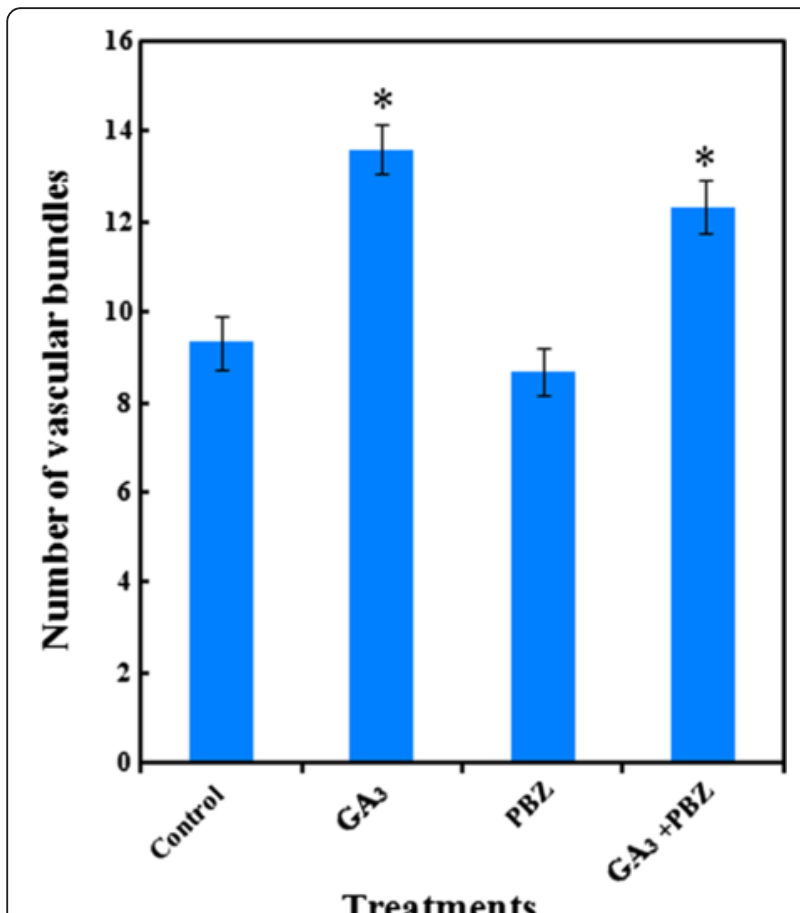

Treatments

Fig. 7 Number of vascular bundles in carrot petioles under different treatments. Data were calculated and presented as mean \pm SD. Student's $t$ test was used to determine the difference between two treatments. ${ }^{*} P<0.05$ was considered to be statistically significant compared with control group
Effects of $\mathrm{GA}_{3}$ application on the expression levels of genes implicated in other hormone pathway

To verify whether $\mathrm{GA}_{3}$ altered metabolism of other hormones, a total of 9 genes from auxin, cytokinin, abscisic acid, brassinolide biosynthetic pathways were identified and their expression under $\mathrm{GA}_{3}$ and PBZ treatments was determined (Table 2; Fig. 11). In carrot roots, transcript levels of most genes were upregulated by $\mathrm{GA}_{3}$ treatment. By contrast, inhibited transcription was detected when PBZ was applied, which was ameliorated by application of $\mathrm{GA}_{3}$. In the petioles, PBZ resulted in obvious increases in transcript levels of DcIPT3, DcABA2, DcMoCo, DcOPR2, DcDAD1, DcDWF4 and DcDWF5, which was quite different from that in the roots. In the leaves, $\mathrm{GA}_{3}$ application increased transcription of DcIPT3, $D c A B A 2$ and $D c D W F 5$ but reduced the mRNA abundance of DcYUCCA, DcCYP83B1, DcOPR2, DcDAD1 and DcDWF4 (Fig. 11).

\section{Discussion}

Modifying plant growth, stature and yield has been a farming goal in professional agronomy and horticulture [27]. The use of plant growth regulators, such as synthetic hormones, has achieved great progress in recent years [28, 29]. GAs, a class of plant hormones, have been widely used to regulate seed germination, plant growth and fruit yield [30-32]. The root crop carrot is popular worldwide and has great value [33]. Thus, regulating the
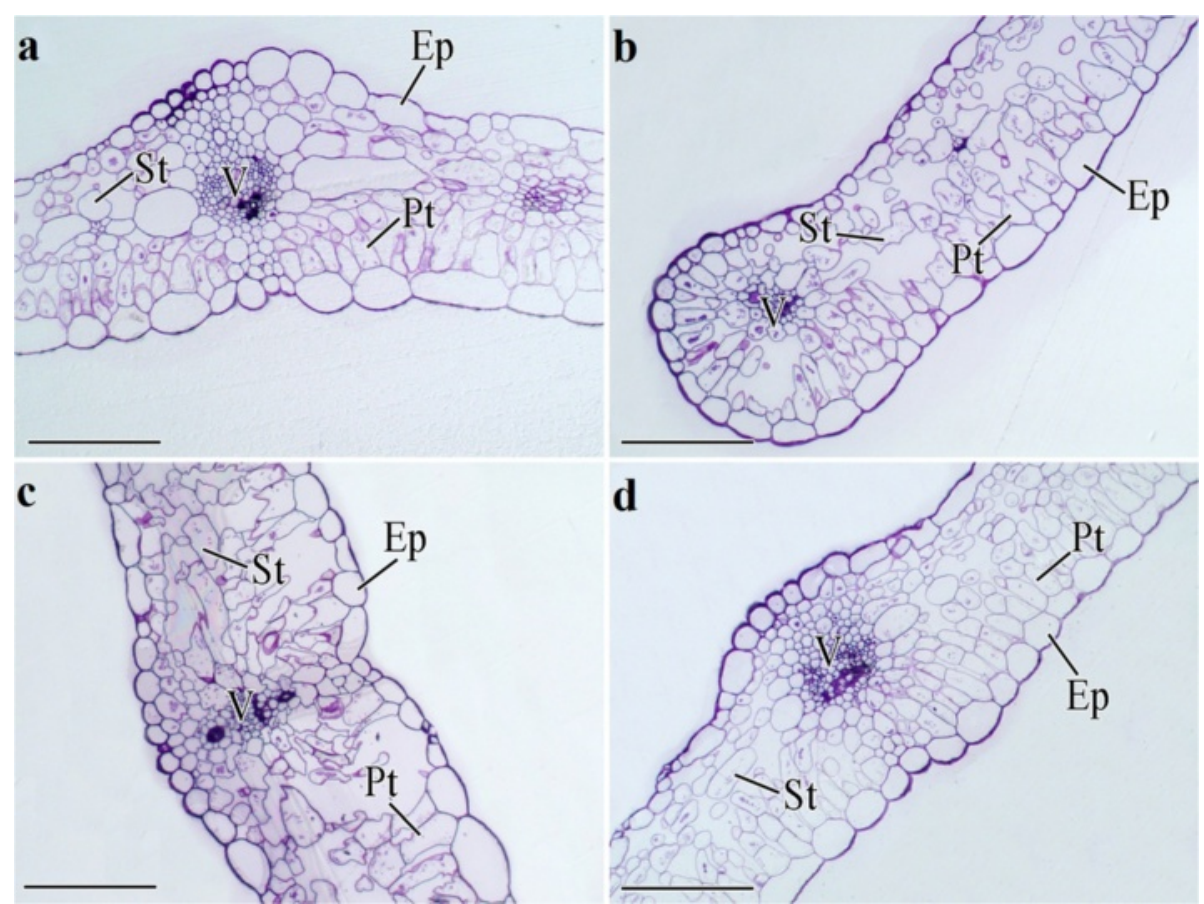

Fig. 8 Effects of $G A_{3}$, PBZ or $G A_{3}+P B Z$ on the anatomical structure in carrot leaves. $\mathbf{a}, \mathbf{b}, \mathbf{c}$ and $\mathbf{d}$ represent the cross sections of leaves under control, $G_{3}, P B Z$ and $G A_{3}+P B Z$ treatments, respectively. Epidermis (Ep), palisade tissue (Pt), spongy tissue (St) and vascular (V) are marked in the figure 
Table 1 Nucleotide sequences of primers specific to gibberellin biosynthetic and signaling genes used for qRT-PCR

\begin{tabular}{|c|c|c|c|}
\hline Gene & Molecular function & $\begin{array}{l}\text { Gene ID in } \\
\text { carrotDB }\end{array}$ & Primer sequences (forward/reverse) \\
\hline DcKS & ent-kaurene synthase & comp49567 & GCGATGGGATGTTGGCGAAGAA/CCGATTGGTGAACTCTGATTGTTGTC \\
\hline DCKO & ent-kaurene oxidase & comp32899 & ATGGTCGCAACAAGTGATTATGATGAG/TCTCTGTTATTACGATGTCGCTTCTGA \\
\hline DCKAO1 & ent-kaurenoic acid oxidase & comp50166 & CACAAGCGGCTGAGACGATTAACA/TTCGACCACTTATCCAATGCAGACTT \\
\hline DcGA200x1 & Gibberellin 20-oxidase & comp575 & CCGACCTCACTCACCATTCTTCAC/CCATCTTGTTCACCACTGCTCTGT \\
\hline DcGA200x2 & Gibberellin 20-oxidase & comp43121 & AACCTAATATCGGATGCTCACAAGTCT/AGGTGGATGAGGTCTTCTTAGTAGAGT \\
\hline DCGA30x1 & Gibberellin 3-oxidase & comp40044 & GGAAGAAATGGGATGGGTCACTGT/CCGTTGGTTAGTATGTGGAGCAGAT \\
\hline DCGA20x1 & Gibberellin 2-oxidase & comp30452 & TTCAGTTCCAGCAGACCAAGACTC/GCTTGAGCAGTGAAGGCAATGG \\
\hline DcGA20x2 & Gibberellin 2-oxidase & comp44237 & TGTTGATGACTGCCTACAGGTAATGAC/CATGAGTGAAGTTGATGGTGCAATCTT \\
\hline DcGA2OX3 & Gibberellin 2-oxidase & comp47688 & ACTTATAATCAGAGCCTGCGAAGAACA/GGAAGGATTGGCGTCAAGTAAGAGAT \\
\hline DcGIDIb & Gibberellin receptor GID1B & comp427507 & ATGCTTCGCCGTCCTGATGG/GCTGACCTATAAACACGATTGAGAAG \\
\hline DcGIDIC & Gibberellin receptor GID1C & comp427506 & AACATGCTTCGCCGTCCTGATG/GAACTGCGTTGGGAGGGACTTTG \\
\hline DCDELLA & DELLA protein GAl & comp43703 & TTGAGCGACACGAGACACTGACT/GAGGTAGCAATAAGCGAGCGAGTG \\
\hline DCSLY1 & F-box protein GID2 & comp28764 & GATAATTTCGCCGACAATTTCGCTGAT/GCCGTCTTGTTCCACTGCTTGT \\
\hline DCPICKLE1 & CHD3-type chromatin-remodeling factor PICKLE & comp46359 & ATGTCCAACTGCTGCTGCTGATAG/TTCCACTTCACAAGATACTGCTTCACA \\
\hline DCPICKLE2 & CHD3-type chromatin-remodeling factor PICKLE & comp48322 & AAGCGAGCTAGAACGAAGACAACC/CGATGGACTGAGTGAGATGAGATGAC \\
\hline DCSPY & $\begin{array}{l}\text { UDP-N-acetylglucosamine-peptide } \\
\text { N-acetylglucosaminyltransferase SPINDLY }\end{array}$ & comp47859 & TGGAGAGTTGGAGTCTGCTATCACT/AATATGCCACGCCTTGGTTAATATCG \\
\hline DCGAMYB & Transcription factor GAMYB & comp43195 & ACTATTCCAGCCAGTTGACTTCTCCT/GCGTCGTCTAATGAACTTCCACTAACA \\
\hline DCSHI & short internodes & comp46084 & GGCAACCAAGCGAAGAAGGATTGTATA/CCAAGAATGTTCACCTGCTGTCTCT \\
\hline
\end{tabular}

root and shoot growth of carrot to increase its yield has been a critical goal. The results of the present study provided new insights into the roles of GA in the growth and development of carrot.

GAs stimulate cell elongation, and this effect has been well studied in GA-deficient mutants [34, 35]. Previous studies on GAs focused on aerial parts because the root is not always economically important. However, the roots of some plants, such as carrot, need intensive attention. The results of the present study showed that $\mathrm{GA}_{3}$ application increased shoot growth but impaired root growth in carrot (Figs. 2 and 3), which is in agreement with the results obtained by Michel-Wolwertz and his collegues [26]. In higher plants, GA could promote cell elongation at the expense of lateral expansion [36]. Here, enlargement of the GA-treated roots was suppressed, again supporting this statement. This treatment also dramatically altered matter distribution. Therefore, excessive $\mathrm{GA}_{3}$ negatively controls root growth in carrot. This interesting observation agrees with a previous research in carrot [26], although some studies indicate that GAs play essential roles in root growth [37, 38].

Xylem is an important tissue for water and solute transport; this tissue also provides structural support [39]. However, the phloem tissue in carrot root provides more nutrients and metabolites than the xylem tissue [40]. As a result, an appropriate phloem/xylem ratio in carrot root is important. A previous study indicated that mobile GA promotes xylem expansion in the hypocotyl of Arabidopsis [41]. Similarly, the present study found that the xylem region in carrot roots treated with $\mathrm{GA}_{3}$ or $\mathrm{GA}_{3}+$ PBZ was evidently multiplied (Fig. 5). This alteration may weaken the root taste, texture and even quality.

GA accumulation within plants may mostly be regulated by biosynthetic genes, and the signals are perceived by receptors and related acting components (Fig. 1) [9, 42]. In the present research, we observed a feedback regulation of GA-related genes. $\mathrm{GA}_{3}$ application decreased the transcript levels of genes encoding GA20-oxidase and GA3oxidase but increased those of genes encoding GA2oxidase, whereas PBZ induced opposite effects on these genes. Indeed, feedback regulation of GA biosynthesis is firmly established as a mechanism to maintain GA homeostasis in higher plants [10, 43].

Treatment with $\mathrm{GA}_{3}$ or its inhibitor PBZ can elevate or reduce GA accumulation, thus exerting different effects on plant growth and development [44, 45]. However, we cannot attribute all the alterations to the changing levels of GAs. Indeed, GA-mediated plant growth often involves complex interaction among hormones [46]. Previous studies revealed that altered GA levels can influence accumulation, signaling, transport and even functions of other hormones [47, 48]. In this study, $\mathrm{GA}_{3}$ induced obvious changes in expression of hormone-related genes, suggesting GAs may interact 


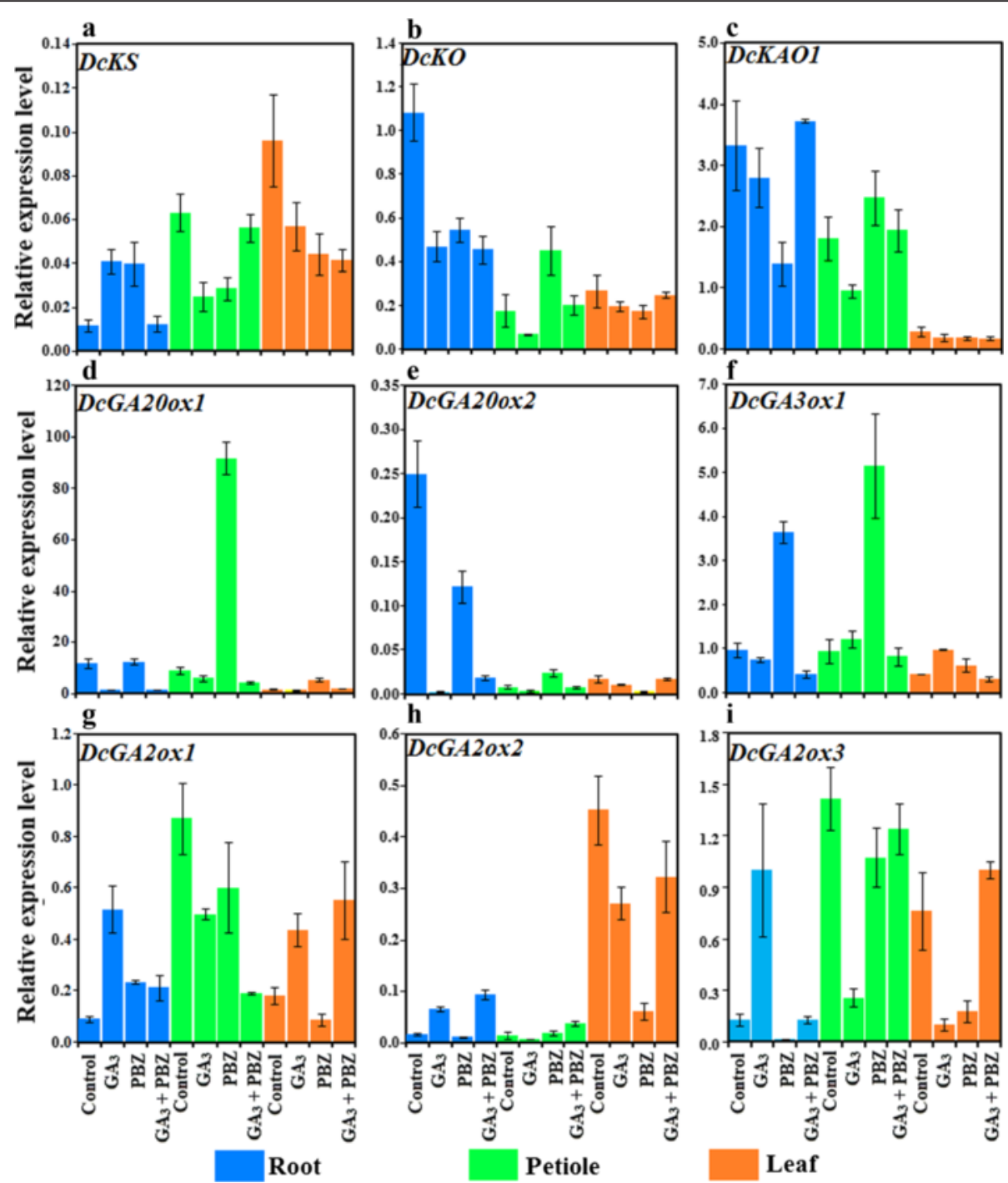

Fig. 9 Effects of $\mathrm{GA}_{3}$, PBZ or $\mathrm{GA}_{3}+\mathrm{PBZ}$ on the expression levels of GA pathway-related genes. Error bars represent standard errors among three independent replicates. Data are the means \pm SD of three replicates

with other hormones to regulate carrot plant growth through crosstalk mechanisms [49-51]. In addition, there seems to be an organ-specific regulation of hormone-related genes in response to $\mathrm{GA}_{3}$ or PBZ. For example, DcGA2ox1 was higher expressed after $\mathrm{GA}_{3}$ treatment in carrot roots and leaves, but was reduced in the petioles. All these results together suggested that hormonal regulation of plant growth is a complicated regulatory network.

\section{Conclusions}

In the present study, $\mathrm{GA}_{3}$ or PBZ treatment altered the morphological parameters, anatomical structure and transcriptional regulatory networks of hormones in carrot plants. $\mathrm{GA}_{3}$ treatment restrained root growth but enhanced shoot growth possibly because of thickened xylem region in the roots and increased area of vascular bundles in the petioles. Excessive or reduced gibberellin also altered hormone homeostasis by changing transcription of related genes, thus exerting effects on carrot plant growth.

\section{Methods}

\section{Plant material and $\mathrm{GA}_{3}$ application}

The seeds of the carrot cultivar 'Kurodagosun' were sown in an artificial chamber at the Nanjing Agricultural University $\left(32^{\circ} 02^{\prime} \mathrm{N}, 118^{\circ} 50^{\prime} \mathrm{E}\right)$. The artificial weather was controlled at $25{ }^{\circ} \mathrm{C}$ for $16 \mathrm{~h}$ during daytime with a light intensity of $300 \mu \mathrm{mol} \mathrm{m} \mathrm{m}^{-2} \mathrm{~s}^{-1}$ followed by $18{ }^{\circ} \mathrm{C}$ for $8 \mathrm{~h}$ at night. Plants were grown in pots $(30 \times 30 \mathrm{~cm})$ filled with a mixture of vermiculite and organic soil (1:1, $\mathrm{v} / \mathrm{v})$. Five weeks after emergence, soils in containers were 

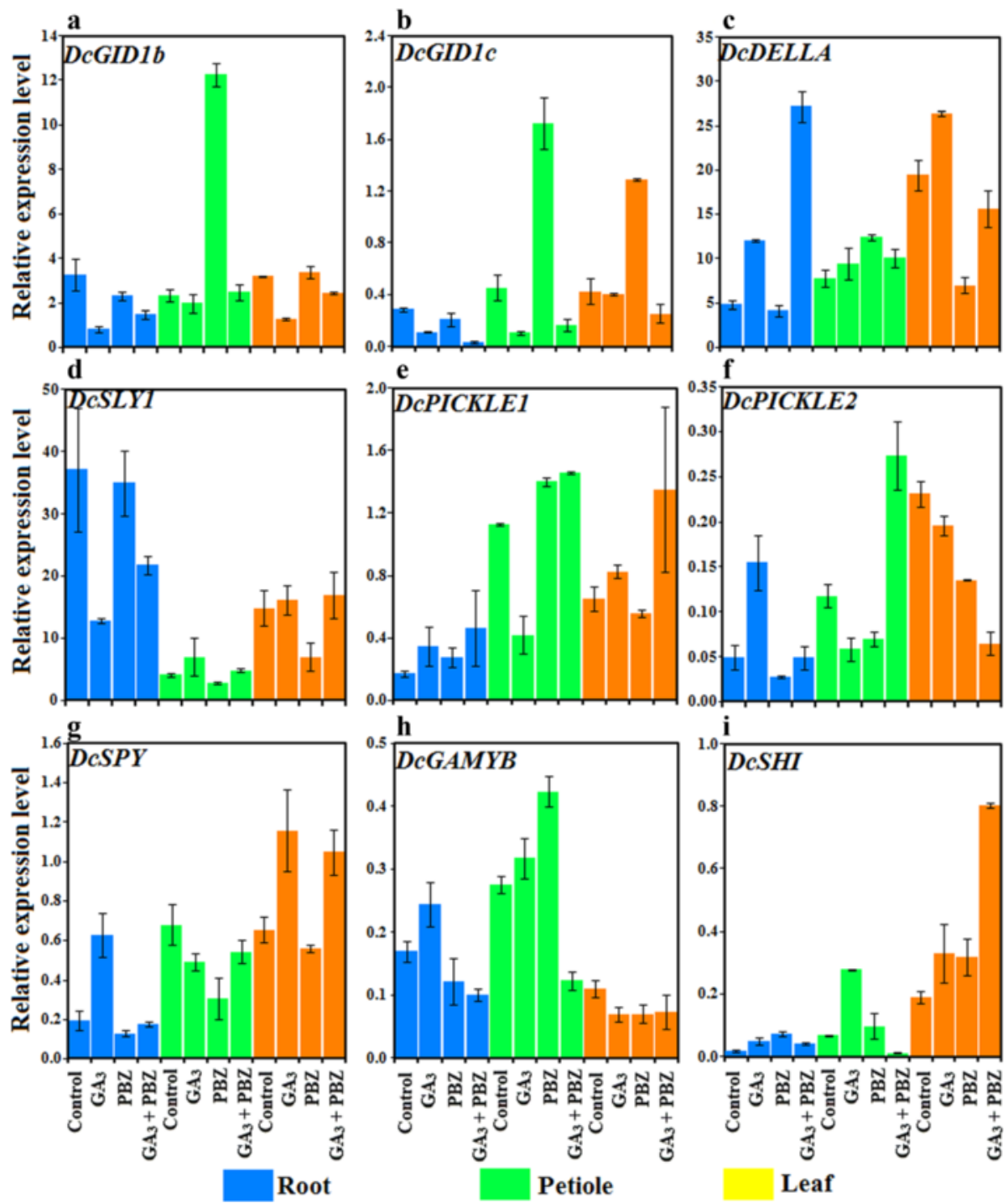

Fig. 10 Effects of $\mathrm{GA}_{3}, \mathrm{PBZ}$ or $\mathrm{GA}_{3}+\mathrm{PBZ}$ on the expression levels of genes involved in GA signaling. Error bars represent the standard errors among three independent replicates. Data are the means \pm SD of three replicates

Table 2 Description of genes implicated in auxin, cytokinin, abscisic acid, brassinolide biosynthesis and primers used for qRT-PCR

\begin{tabular}{llll}
\hline Gene & Molecular function & Gene ID in carrotDB & Primer sequences (forward/reverse) \\
\hline DCYUCCA & Indole-3-pyruvate monooxygenase & comp48938 & GTTCTGTCCAGTCCGAGGTTGAG/CCTCTCCTCCGAACTCTTGTAATCC \\
DcCYP83B1 & Cytochrome P450 83B1 & comp43558 & GACATGCTGAGTACGGCAGTTACC/TCCAATGAATGGAAGTCCAGGAGGA \\
DCIPT3 & Adenylate isopentenyltransferase & comp34617 & GAATGGAATGGTAGATGAGGCAAGACA/TCTCTAACTGGCGGCAGGCTAG \\
DCABA2 & Xanthoxin dehydrogenase & comp50471 & CGATATTATGGTCAACAATGCGGGTAT/CGTCGTGCTGCTTACACTACTCA \\
DCMoCo & MoCo sulfurase & comp15442 & CCTGGAACTGATTGGAATACCGAAGT/GCCTGGATTAATGGAATAGACGCCTTA \\
DCOPR2 & OPDA reductase & comp45214 & CACATGGTGGAGCCAAGGATGAA/AGCAACAAGGTCAGTACAGTTCTCAG \\
DCDAD1 & Lipase & comp46390 & CTCGAAGAGGAGCTTAGTGAAGTTTT/TAGGAAGAAGAAGGACTCGGCAATAC \\
DCDWF4 & Cytochrome P450 90B1 & comp42688 & AAACGCTAAGGCTGGGCAATGT/GCACGGCTGCAATCACTGGAA \\
DCDWF5 & Sterol delta-7 reductase & comp15451 & AGATGGTGGTGAAGGAGGAGAA/CACGCAGTCATAGTGGGTITG \\
\hline
\end{tabular}



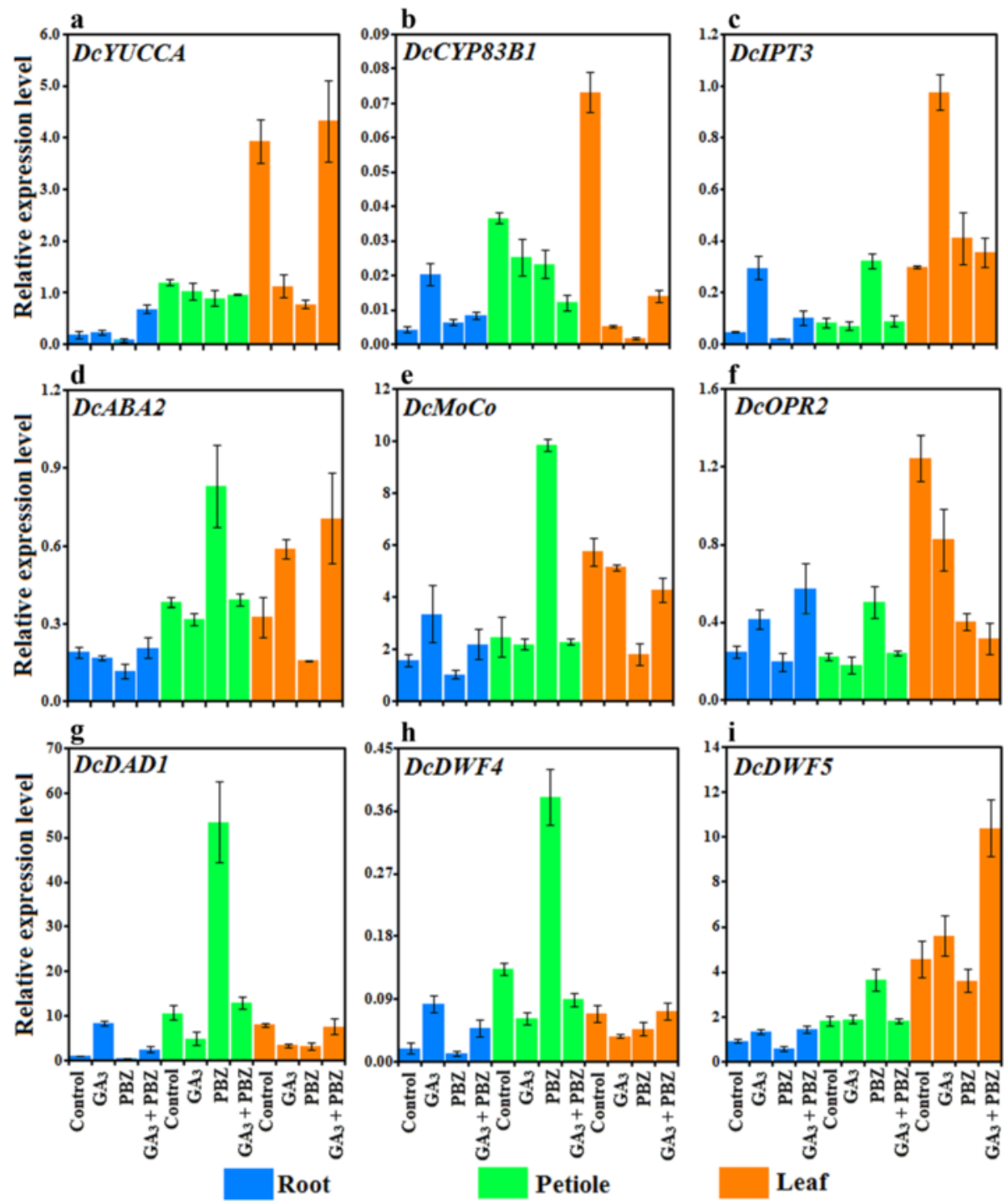

Fig. 11 Effects of $\mathrm{GA}_{3}, \mathrm{PBZ}$ or $\mathrm{GA}_{3}+\mathrm{PBZ}$ on the expression levels of genes involved in auxin, cytokinin, abscisic acid and brassinolide metabolism. Error bars represent the standard errors among three independent replicates. Data are the means \pm SD of three replicates

irrigated with $200 \mathrm{~mL}$ of aqueous solution that contains $\mathrm{GA}_{3}$ (150 ppm), PBZ (20 ppm), or the combination of both. The plants treated with aqueous solution were used as the control. All the treatments were performed weekly until the fifth week. The samples were replicated three times and harvested after the treatments. Then, the samples were morphologically characterized before storing at $-80{ }^{\circ} \mathrm{C}$ until analysis.

\section{Anatomical structure analysis}

To examine the effects of $\mathrm{GA}_{3}$ or PBZ treatment on carrot growth, the anatomical structure of the plants was investigated. Fresh samples were cut into small pieces of approximately $1 \mathrm{~mm}^{3}$ and then immediately stored in phosphate buffer solution ( $\mathrm{pH} 7.2$ ) containing $2.5 \%$ glutaraldehyde. The slices were dehydrated with gradient ethanol and then infiltrated with epoxy propane. For embedding, the samples were placed and soaked in Spurr resin [52]. A Leica ultramicrotome (Germany) was used to cut the samples into thin sections $(\sim 1 \mu \mathrm{m})$. The sections were then stained with $0.5 \%$ methyl violet for $10 \mathrm{~min}$. Subsequently, the slices were placed under a Leica DMLB microscope (Germany) for observation and taking photographs.

\section{Total RNA isolation and cDNA synthesis}

Total RNA was strictly extracted from carrot roots, petioles and leaves using an RNA extraction kit (Tiangen, Beijing, China) in accordance with the manufacturer's directions. RNA was quantified by a One-Drop ${ }^{\text {Tw }}$ spectrophotometer. 
Total RNA was treated with gDNA Eraser for 2 min at $42{ }^{\circ} \mathrm{C}$ (TaKaRa, Dalian, China) to eliminate genomic DNA contaminants. First-strand cDNA was synthesized from the isolated RNA using a PrimeScript RT reagent kit (TaKaRa, Dalian, China) in accordance with the manufacturer's specifications. The cDNA reaction mixture was diluted 10-fold in deionized water for qRT-PCR analysis.

\section{Gene expression analysis by quantitative real-time PCR}

Genes involved in GA, auxin, cytokinin, abcisic acid and brassinolide pathways were selected from carrotDB (http:// apiaceae.njau.edu.cn/carrotdb/index.php) [23]. qRT-PCR was performed using TaKaRa SYBR Premix Ex Taq (Takara, Dalian, China) in a total of volume of $20 \mu \mathrm{L}$. All PCR reaction mixtures contained $10 \mu \mathrm{L}$ of SYBR Premix Ex Taq, $7.4 \mu \mathrm{L}$ of deionized water, $0.4 \mu \mathrm{L}$ of each forward and reverse primer, and $2 \mu \mathrm{L}$ of diluted cDNA strand. PCR cycling was performed using a program of $95^{\circ} \mathrm{C}$ for $30 \mathrm{~s}$, followed by 40 cycles of $95{ }^{\circ} \mathrm{C}$ for $5 \mathrm{~s}$ and $60^{\circ} \mathrm{C}$ for $30 \mathrm{~s}$. The experiments were performed with three independent biological replicates and the results were normalized against carrot reference gene DcACTIN [53]. Data from $D c G A 2 o x 3$ in the leaves of carrot grown under GA + PBZ treatment were selected as calibrator for gene expression analysis. The PCR primer pairs of all genes are shown in Tables 1 and 2.

\section{Statistical analysis}

Student $t$ test was applied to detect differences under different treatments at the 0.05 significance level.

\section{Availability of supporting data}

The data supporting the results of this article are included within the article.

\section{Abbreviations \\ C: collenchyma; CPS: ent-copalyl diphosphate synthase; Ep: epidermis; Ex: exodermis; GA: gibberellin; GA20ox: GA20-oxidase; GA2ox: GA2-oxidase; GA3ox: GA3-oxidase; GGDP: geranyl geranyl diphosphate; GID1: gibberellin Insensitive Dwarf1; KAO: ent-kaurenoic acid oxidase; KO: ent-kaurene oxidase; KS: ent-kaurene synthase; P: phloem; PBZ: paclobutrazol; PC: parenchymal cell; Ph: phellogen; Pt: palisade tissue; qRT-PCR: quantitative real-time PCR; SG: starch granule; SHI: short internode; SLY1: sleepy1; SPY: spindly; St: spongy tissue; $V$ : vascular; Ve: vessel; $X$ : xylem.}

\section{Competing interests}

The authors declare that there are no competing interests.

\section{Authors' contributions}

Conceived and designed the experiments: ASX GLW. Performed the experiments: GLW FQ. Analyzed the data: GLW ZSX FW. Contributed reagents/materials/analysis tools: ASX. Wrote the paper: GLW. Revised the paper: GLW ASX. All authors read and approved the final manuscript.

\section{Acknowledgements}

The research was supported by the New Century Excellent Talents in University (NCET-11-0670); Jiangsu Natural Science Foundation (BK20130027); Shanxi Province Coal Based Key Scientific and Technological Project (FT201402-07); Priority Academic Program Development of Jiangsu Higher Education Institutions.
Received: 17 August 2015 Accepted: 7 December 2015

Published online: 15 December 2015

\section{References}

1. Malamy JE. Intrinsic and environmental response pathways that regulate root system architecture. Plant Cell Environ. 2005;28(1):67-77.

2. Wolters $H_{\text {, Jurgens }} \mathrm{G}$. Survival of the flexible: hormonal growth control and adaptation in plant development. Nat Rev Genet. 2009;10(5):305-17.

3. Pimenta Lange MJ, Lange T. Gibberellin biosynthesis and the regulation of plant development. Plant Biol. 2006;8(3):281-90.

4. Hu J, Mitchum MG, Barnaby N, Ayele BT, Ogawa M, Nam E, et al. Potential sites of bioactive gibberellin production during reproductive growth in Arabidopsis. Plant Cell. 2008;20(2):320-36.

5. Claeys H, De Bodt S, Inzé D. Gibberellins and DELLAs: central nodes in growth regulatory networks. Trends Plant Sci. 2014;19(4):231-9.

6. Yamaguchi S. Gibberellin metabolism and its regulation. Annu Rev Plant Biol. 2008;59(1):225-51.

7. Sun TP. Gibberellin-GID1-DELLA: a pivotal regulatory module for plant growth and development. Plant Physiol. 2010;154(2):567-70.

8. Murase K, Hirano Y, Sun TP, Hakoshima T. Gibberellin-induced DELLA recognition by the gibberellin receptor GID1. Nature. 2008;456(7221):459-63.

9. Olszewski N, Sun TP, Gubler F. Gibberellin signaling: biosynthesis, catabolism, and response pathways. Plant Cell. 2002;14 suppl 1:S61-80.

10. Israelsson M, Mellerowicz E, Chono M, Gullberg J, Moritz T. Cloning and overproduction of gibberellin 3-oxidase in hybrid aspen trees. Effects on gibberellin homeostasis and development. Plant Physiol. 2004;135(1):221-30.

11. Ueguchi-Tanaka M, Ashikari M, Nakajima M, Itoh H, Katoh E, Kobayashi M, et al. GIBBERELLIN INSENSITIVE DWARF1 encodes a soluble receptor for gibberellin. Nature. 2005;437(7059):693-8.

12. Carrera E, Bou J, García-Martínez JL, Prat S. Changes in GA 20-oxidase gene expression strongly affect stem length, tuber induction and tuber yield of potato plants. Plant J. 2000;22(3):247-56.

13. Debeaujon I, Koornneef M. Gibberellin requirement for Arabidopsis seed germination is determined both by testa characteristics and embryonic abscisic Acid. Plant Physiol. 2000;122(2):415-24.

14. Kassen CM, Zagorski S, Kepczynski J, Groot SPC. Key role for endogenous gibberellins in the control of seed germination. Ann Bot. 1989;63(1):71-80.

15. Eriksson S, Böhlenius $\mathrm{H}$, Moritz T, Nilsson O. GA $\mathrm{A}_{4}$ Is the active gibberellin in the regulation of $L E A F Y$ transcription and Arabidopsis floral initiation. Plant Cell. 2006;18(9):2172-81.

16. Serrani JC, Sanjuán R, Ruiz-Rivero O, Fos M, García-Martínez JL. Gibberellin regulation of fruit set and growth in tomato. Plant Physiol. 2007;145(1):246-57.

17. Rebers M, Kaneta $T$, Kawaide $H$, Yamaguchi S, Yang YY, Imai $R$, et al. Regulation of gibberellin biosynthesis genes during flower and early fruit development of tomato. Plant J. 1999;17(3):241-50.

18. Gou J, Strauss SH, Tsai CJ, Fang K, Chen Y, Jiang X, et al. Gibberellins regulate lateral root formation in Populus through interactions with auxin and other hormones. Plant Cell. 2010:22(3):623-39.

19. Ubeda-Tomás S, Federici F, Casimiro I, Beemster GTS, Bhalerao R, Swarup R, et al. Gibberellin signaling in the endodermis controls Arabidopsis root meristem size. Curr Biol. 2009;19(14):1194-9.

20. Wang GL, Xu ZS, Wang F, Li MY, Tan GF, Xiong AS. Regulation of ascorbic acid biosynthesis and recycling during root development in carrot (Daucus carota L.). Plant Physiol Biochem. 2015:94:10-8.

21. Luby CH, Maeda HA, Goldman IL. Genetic and phenological variation of tocochromanol (vitamin E) content in wild (Daucus carota L. var. carota) and domesticated carrot (D. carota L. var. sativa). Hortic Res. 2014;1:14015.

22. Xu ZS, Huang Y, Wang F, Song X, Wang GL, Xiong AS. Transcript profiling of structural genes involved in cyanidin-based anthocyanin biosynthesis between purple and non-purple carrot (Daucus carota L.) cultivars reveals distinct patterns. BMC Plant Biol. 2014;14(1):262.

23. Xu ZS, Tan HW, Wang F, Hou XL, Xiong AS. CarrotDB: a genomic and transcriptomic database for carrot. Database. 2014;2014:bau096.

24. Maloof JN. Plant development: slowing root growth naturally. Curr Biol. 2004;14(10):R395-6.

25. Mitsuhashi W, Toyomasu T, Masui H, Katho T, Nakaminami K, Kashiwagi Y, et al. Gibberellin is essentially required for carrot (Daucus carota L.) somatic embryogenesis: dynamic regulation of gibberellin 3-oxidase gene expressions. Biosci Biotechnol Biochem. 2003;67(11):2438-47. 
26. Michel-Wolwertz MR, Sironval C. Inhibition of growth and accumulation of B-carotene in carrot roots by gibberellic acid. Phytochemistry. 1963;2(2):183-7.

27. Biemelt S, Tschiersch H, Sonnewald U. Impact of altered gibberellin metabolism on biomass accumulation, lignin biosynthesis, and photosynthesis in transgenic tobacco plants. Plant Physiol. 2004;135(1):254-65.

28. Cai T, Xu H, Peng D, Yin Y, Yang W, Ni Y, et al. Exogenous hormonal application improves grain yield of wheat by optimizing tiller productivity. Field Crop Res. 2014;155:172-83.

29. Gray WM. Hormonal regulation of plant growth and development. PLoS Biol. 2004;2(9):e311.

30. Peng J, Harberd NP. The role of GA-mediated signalling in the control of seed germination. Curr Opin Plant Biol. 2002;5(5):376-81.

31. de Souza IRP, MacAdam JW. Gibberellic acid and dwarfism effects on the growth dynamics of B73 maize (Zea mays L.) leaf blades: a transient increase in apoplastic peroxidase activity precedes cessation of cell elongation. J Exp Bot. 2001;52(361):1673-82.

32. Southwick SM, Glozer K. Reducing flowering with gibberellins to increase fruit size in stone fruit trees: applications and implications in fruit production. HortTechnol. 2000;10(4):744-51.

33. Just BJ, Santos CAF, Fonseca MEN, Boiteux LS, Oloizia BB, Simon PW. Carotenoid biosynthesis structural genes in carrot (Daucus carota): isolation, sequence-characterization, single nucleotide polymorphism (SNP) markers and genome mapping. Theor Appl Genet. 2007;114(4):693-704.

34. Weier D, Thiel J, Kohl S, Tarkowská D, Strnad M, Schaarschmidt S, et al. Gibberellin-to-abscisic acid balances govern development and differentiation of the nucellar projection of barley grains. J Exp Bot. 2014;65(18):5291-304.

35. Willige BC, Ghosh S, Nill C, Zourelidou M, Dohmann EMN, Maier A, et al. The DELLA domain of GA INSENSITIVE mediates the interaction with the GA INSENSITIVE DWARF1A gibberellin receptor of Arabidopsis. Plant Cell. 2007;19(4):1209-20.

36. Eriksson ME, Israelsson M, Olsson O, Moritz T. Increased gibberellin biosynthesis in transgenic trees promotes growth, biomass production and xylem fiber length. Nat Biotechnol. 2000;18(7):784-8.

37. Fu X, Harberd NP. Auxin promotes Arabidopsis root growth by modulating gibberellin response. Nature. 2003:421(6924):740-3.

38. Achard P, Cheng H, De Grauwe L, Decat J, Schoutteten H, Moritz T, et al. Integration of plant responses to environmentally activated phytohormonal signals. Science. 2006;311(5757):91-4.

39. Demura T, Ye ZH. Regulation of plant biomass production. Curr Opin Plant Biol. 2010;13(3):298-303.

40. Arscott SA, Tanumihardjo SA. Carrots of many colors provide basic nutrition and bioavailable phytochemicals acting as a functional food. Compr Rev Food Sci Food Saf. 2010;9(2):223-39.

41. Ragni L, Nieminen K, Pacheco-Villalobos D, Sibout R, Schwechheimer C, Hardtke CS. Mobile gibberellin directly stimulates Arabidopsis hypocotyl xylem expansion. Plant Cell. 2011;23(4):1322-36.

42. Hedden P. Recent advances in gibberellin biosynthesis. J Exp Bot. 1999; 50(334):553-63.

43. Gallego-Giraldo L, Ubeda-Tomás S, Gisbert C, García-Martínez JL, Moritz T, López-Díaz I. Gibberellin homeostasis in tobacco is regulated by gibberellin metabolism genes with different gibberellin sensitivity. Plant Cell Physiol. 2008:49(5):679-90.

44. Saos FLGL, Hourmant A, Esnault F, Chauvin JE. In vitro bulb development in shallot (Allium cepa L. Aggregatum Group): effects of anti-gibberellins, sucrose and light. Ann Bot. 2002;89(4):419-25.

45. Eshel D, Lichter A, Dinoor A, Prusky D. Characterization of Alternaria alternata glucanase genes expressed during infection of resistant and susceptible persimmon fruits. Mol Plant Pathol. 2002;3(5):347-58.

46. Gao XH, Xiao SL, Yao QF, Wang YJ, Fu XD. An updated GA signaling 'relief of repression' regulatory model. Mol Plant. 2011:4(4):601-6.

47. Oh E, Yamaguchi S, Hu J, Yusuke J, Jung B, Paik I, et al. PIL5, a phytochrome-interacting Bhlh protein, regulates gibberellin responsiveness by binding directly to the GAl and RGA promoters in Arabidopsis seeds. Plant Cell. 2007;19(4):1192-208.

48. Willige BC, Isono E, Richter R, Zourelidou M, Schwechheimer C. Gibberellin regulates PIN-FORMED abundance and is required for auxin transportdependent growth and development in Arabidopsis thaliana. Plant Cell. 2011;23(6):2184-95.
49. Yang C, Liu J, Dong X, Cai Z, Tian W, Wang X. Short-term and continuing stresses differentially interplay with multiple hormones to regulate plant survival and growth. Mol Plant. 2014;7(5):841-55.

50. Liu J, Rowe J, Lindsey K. Hormonal crosstalk for root development: a combined experimental and modeling perspective. Front Plant Sci. 2014;5:116.

51. Nemhauser JL, Hong F, Chory J. Different plant hormones regulate similar processes through largely nonoverlapping transcriptional responses. Cell. 2006;126(3):467-75.

52. Spurr AR. A low-viscosity epoxy resin embedding medium for electron microscopy. J Ultrastruct Res. 1969;26(1-2):31-43.

53. Tian C, Jiang Q, Wang F, Wang GL, Xu ZS, Xiong AS. Selection of suitable reference genes for $\mathrm{PPCR}$ normalization under abiotic stresses and hormone stimuli in carrot leaves. PLoS One. 2015:10(2):e0117569.

\section{Submit your next manuscript to BioMed Central and we will help you at every step:}

- We accept pre-submission inquiries

- Our selector tool helps you to find the most relevant journal

- We provide round the clock customer support

- Convenient online submission

- Thorough peer review

- Inclusion in PubMed and all major indexing services

- Maximum visibility for your research

Submit your manuscript at www.biomedcentral.com/submit 